\title{
Ring-based interleaver for Nyquist filtering and WDM multiplexing
}

\author{
Leimeng Zhuang ${ }^{1, *}$, Benjamin Foo ${ }^{1}$, Chen Zhu ${ }^{1}$, Bill Corcoran ${ }^{1,2}$, Binhuang Song ${ }^{1}$, and Arthur J. Lowery ${ }^{1,2}$ \\ Electro-Photonics Laboratory, Electrical and Computer Systems Engineering, Monash University, Clayton, VIC3800, Australia \\ ${ }^{2}$ Centre for Ultrahigh-bandwidth Devices for Optical Systems (CUDOS), Australia \\ *leimeng.zhuang@monash.edu
}

\begin{abstract}
We analyze the system performance of a novel ring-resonator circuit for simultaneous Nyquist pulse shaping and (de)multiplexing, which features a roll-off factor of 0.025 and is able to dramatically reduce the digital signal processing workload.

OCIS codes: (060.2330) Fiber optics communications; (060.1660) coherent communications; (250.3140) Integrated optoelectronic circuits; (120.2440) Filter; (320.5540) Pulse shaping; (060.4230) Multiplexing.
\end{abstract}

\section{Introduction}

Recently Nyquist wavelength division multiplexing (WDM) transmission systems have been proposed and demonstrated with very high spectral efficiencies. Compared with orthogonal frequency division multiplexing (OFDM), Nyquist WDM (N-WDM) has lower receiver bandwidth requirement for achieving the same performance [1]. The Nyquist filter that generates the rectangular-shaped signal spectrum is usually implemented at the transmitter end and can be achieved in either the electrical or optical domains. Digital filtering is flexible which provides near optimal filter profile, but it requires heavy computational effort and the maximum producible symbol rate is limited by the sampling speed of the state-of-the-art digital-to-analog converter [2]. Optical filtering, on the other hand, does not have this limiting factor. A good filter shape, for example, can be achieved by means of a commercial waveshaper based on liquid crystal on silicon (LCoS) technology [3] or a dedicated arrayed waveguide grating router-assisted waveshaping subsystem featuring fine resolution [4]. However, it is still a challenge to design an optical Nyquist filter featuring a very small roll-off factor.

We show that a waveguide frequency-interleaver circuit based on a 3-ring resonator-assisted Mach-Zehnder interferometer (3RAMZI) structure can be used, giving the possibility of miniaturization using planar optical integrated circuit technology. Using theoretical analysis and numerical simulations, we demonstrate that our circuit is able to achieve high spectral-efficient optical Nyquist filtering with a small roll-off factor of 0.025 and $25-\mathrm{dB}$ stopband attenuation, with each passband having a spectral occupation of $50 \%$ of the device free spectral range (FSR), as well as flat magnitude and group delay profiles. Secondly, its interleaving and switching functionalities allow two sets of equally-spaced WDM channels (even and odd numbered channels) to be (de)multiplexed according to an adaptive channel routing plan. This can also be utilized in combination with a waveshaper to enable a flexible, fine-resolution add-drop multiplexing functionality. Furthermore, when equipped with full tunablility, the circuit can be reconfigured to provide a variety of other filter shapes and functionalities [5], which are of significant use for the construction of on-chip programmable photonic signal processors.

\section{Device principle}

Figure 1 shows a schematic of the frequency-interleaver circuit. Two ring resonators are coupled to the shorter arm of the MZI in the cascaded manner and a third resonator is coupled to the longer arm. All three resonators have a circumference equal to twice the difference in lengths of the two MZI arms, $\Delta L$. The ring parameters $\kappa_{\mathrm{n}}$ and $\phi_{\mathrm{n}}$ express the optical power coupling coefficients between the ring and MZI arms, and additional optical phase shifts, in the rings. The ring to MZI arm transfer function, $\mathbf{H}$, can be derived from a digital filter model in z-transform [6]:

with

$$
\begin{gathered}
\mathbf{H}=\left[\begin{array}{ll}
H_{11} & H_{12} \\
H_{21} & H_{22}
\end{array}\right]=\eta\left[\begin{array}{cc}
\sqrt{1-\kappa_{\mathrm{R}}} & -\mathrm{j} \sqrt{\kappa_{\mathrm{R}}} \\
-\mathrm{j} \sqrt{\kappa_{\mathrm{R}}} & \sqrt{1-\kappa_{\mathrm{R}}}
\end{array}\right]\left[\begin{array}{cc}
A_{\mathrm{U}}(z) & 0 \\
0 & A_{\mathrm{L}}(z)
\end{array}\right]\left[\begin{array}{cc}
\sqrt{1-\kappa_{\mathrm{L}}} & -\mathrm{j} \sqrt{\kappa_{\mathrm{L}}} \\
-\mathrm{j} \sqrt{\kappa_{\mathrm{L}}} & \sqrt{1-\kappa_{\mathrm{L}}}
\end{array}\right] \\
A_{\mathrm{U}}(z)=\frac{\sqrt{1-\kappa_{1}}-t^{2} z^{-2} e^{-\mathrm{j} \phi_{1}}}{1-\sqrt{1-\kappa_{1}} t^{2} z^{-2} e^{-\mathrm{j} \phi_{1}}} \cdot \frac{\sqrt{1-\kappa_{2}}-t^{2} z^{-2} e^{-\mathrm{j} \phi_{2}}}{1-\sqrt{1-\kappa_{2}} t^{2} z^{-2} e^{-\mathrm{j} \phi_{2}}} \\
A_{\mathrm{L}}(z)=\frac{\sqrt{1-\kappa_{3}}-t^{2} z^{-2} e^{-\mathrm{j} \phi_{3}}}{1-\sqrt{1-\kappa_{3}} t^{2} z^{-2} e^{-\mathrm{j} \phi_{3}} \cdot t z^{-1} e^{-\mathrm{j} \phi_{\mathrm{D}}}}
\end{gathered}
$$$$
\text { and }
$$

where $z=\exp (-\mathrm{j} v)$ with $v=[-\pi, \pi]$ representing the angular frequency normalized to the free spectral range (FSR) of the device $\left(\Delta f_{\mathrm{FSR}}=1 / \Delta T=c /\left(\Delta L \cdot n_{\mathrm{g}}\right)\right.$ with $\Delta T$ the delay time for an optical path of $\Delta L, c$ the vacuum speed of light and $n_{\mathrm{g}}$ group index of the waveguide); $t$ is the amplitude transmission coefficient for an optical path of $\Delta L$ 
(determined by the waveguide loss), and $\eta$ is a complex coefficient which accounts for the general insertion loss and phase shift introduced by the MZI. Using Eqs. (1)-(3), we derive the frequency responses between different input and output ports $H_{\mathrm{mn}}(m, n=[1,2])$. Attributed to the MZI structure, the bar- and cross-port transfer functions are a pair of complementary spectral filters. In terms of the digital filter concept, the filters feature seven roots in their numerators and six in their denominators, namely seven zeros and six poles [6]. By properly setting the circuit parameters as shown in Fig. 1a, we can configure the positions of the zeros and poles in the polar plane such that the circuit synthesizes a $7^{\text {th }}$-order IIR Chebyshev Type II filter, which is characterized by flat passband and equal-ripple stopband as shown in Fig. 1b. Based on this type of filter, a good frequency-interleaver with nearly rectangularshaped transmission profiles can be achieved. Notably, the transition bands for the transmission dropping from -3 to $-25 \mathrm{~dB}$ are as small as $1 \% \mathrm{FSR}$, meaning a sharp roll-off relative to passbands with a bandwidth of $50 \%$ FSR. In the context of N-WDM, this closely approximates a root raised cosine (RRC) digital Nyquist filter with a roll-off factor of 0.025 at $-25 \mathrm{~dB}$, whose generation requires a number of 236 filter taps. A filter shape comparison between the is shown in Fig. 1c. In the above calculations, the waveguide loss is $0.1 \mathrm{~dB} / \Delta L$, or equivalently $0.2 \mathrm{~dB}$ per roundtrip in the ring resonator, which is within the capability of the current waveguide technologies [7]. The achieved performance does not require $\kappa_{\mathrm{n}}$ approaching 1 nor $\phi_{\mathrm{n}}$ much greater than $\pi$ that may raise difficult challenges for device realization. Using the waveguide technology in [5], for example, and implementing $\kappa_{\mathrm{n}}$ and $\phi_{\mathrm{n}}$ by means of MZI-based tunable couplers and thermo-optical index variations, all the desired circuit parameters fall within the typical tuning/trimming ranges limited by the fabrication tolerances and device electrical-power-handling capacity.
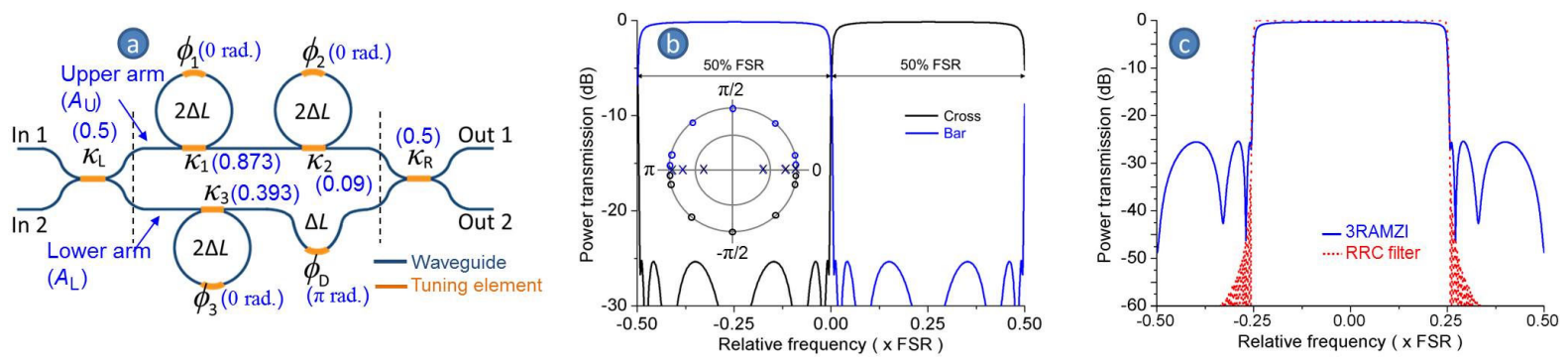

Fig. 1: (a) schematic of a 3RAMZI circuit and a set of circuit parameters to achieve a rectangular-shaped filter profile; (b) complementary transmissions between bar and cross ports and the corresponding zero-pole diagram; (c) comparison to a root raise cosine (RRC) digital Nyquist filter with a roll-off factor of 0.025 at $-25 \mathrm{~dB}$.

\section{WDM transmission investigation}

To investigate the WDM transmission performance based on the 3RAMZI circuit, the system as depicted in Fig. 2a is simulated, where seven PDM-QPSK N-WDM channels are generated using the 3RAMZI circuit as Nyquist pulse shaping filter. As a numerical example, the digitally generated QPSK symbols of each transmitter are modulated on a CW light at the rate of 20 Gbaud. The WDM channel spacing is set at $20 \mathrm{GHz}$. The even and odd channels are multiplexed separately and fed as two channel groups to the two inputs of the 3RAMZI. Accordingly, the 3RAMZI is designed to have a FSR of $40 \mathrm{GHz}$, yielding an interleaver channel bandwidth and spacing of $20 \mathrm{GHz}$. In this case, all the WDM channels are Nyquist-shaped simultaneously using only a single device, however, the channel spacing is fixed equal to the channel bandwidth due to the interleaver property as shown in Fig. 1b, leaving no flexibility for guard band (GB) insertion. Alternatively, the 3RAMZI can also be used as a single-channel Nyquist shaping filter, with one being placed in each WDM channel before multiplexing. This allows the WDM channel spacing to be adjusted freely for GB insertion; however, it will incur an increase in system complexity.

Figure $2 \mathrm{~b}$ to $2 \mathrm{e}$ shows the calculated error vector magnitude (EVM) of the center channel based on back-toback transmission versus optical signal-to-noise ratio (OSNR) in a $0.1 \mathrm{~nm}$ optical bandwidth. In the simulation, a $30-\mathrm{GHz}$ bandwidth optical bandpass filter (OBPF) is used for channel selection, and then a fractionally spaced adaptive CMA filter with additional taps is used to achieve convergence to the optimal matched filter performance [8]. Figure $2 \mathrm{~b}$ compares the system performance of the 3RAMZI interleaver with that of the digital RRC filter counterpart (Fig. 1c). When the systems operate in the noise-limited regime with relatively low OSNR, the interchannel crosstalk due to the non-zero Nyquist filter roll-off plays a minor role. Notably, a penalty of $2 \mathrm{~dB}$ is observed for the 3RAMZI as compared to the digital RRC filter. We attribute this to the difference in the filter stopband suppression as shown in Fig. 1c. At high OSNRs, however, the performance difference becomes smaller. This is because the crosstalk becomes the dominant impairment and is dependent on the filter roll-off factor.

Figure 2c compares the transmission performance of a 3RAMZI used as the receiver optical matched filter with that of the $30-\mathrm{GHz}$ standard OBPF plus digital matched filter solution. Here, the N-WDM channels are 
generated using the digital Nyquist filter approach. We note that with the 3RAMZI-based optical matched filtering, only a few taps is required for the adaptive equalizer to adjust the sampling phase. Notably, to achieve the optimal performance, 3RAMZI required only 9 digital filter taps while the standard digital matched filter approach required 30 taps. This advantage is further demonstrated in Fig. $2 \mathrm{~d}$ showing that when an optical matched filter is used, a lower number of digital filter taps are required to reach optimal performance.

Figure 2e demonstrates the feasibility of using 3RAMZI as both Nyquist shaping filter and optical matched filter simultaneously. Compared with using digtal RRC filter at both transmitter and receiver, the 3RAMZI-based all-optical solution has an OSNR penalty of about $1 \mathrm{~dB}$ at the FEC threshold, and this penalty becomes smaller as the OSNR increases. Within the fabrication capability, 3RAMZI can be scaled to arbitrary baud rate without causing performance degradation, which is shown in Fig. 2e by a comparison between a 20-Gbaud and a 10-Gbaud case.

(a)
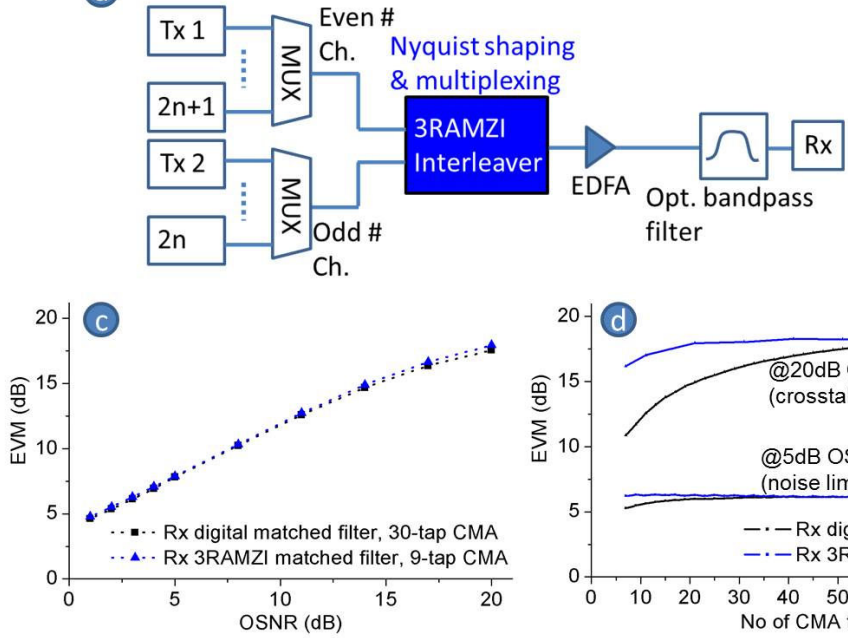

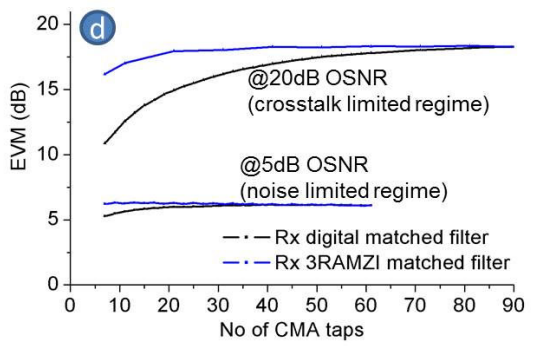

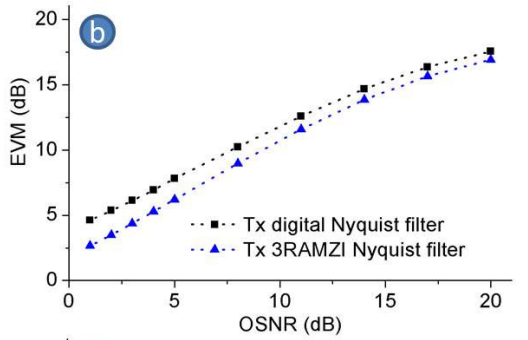

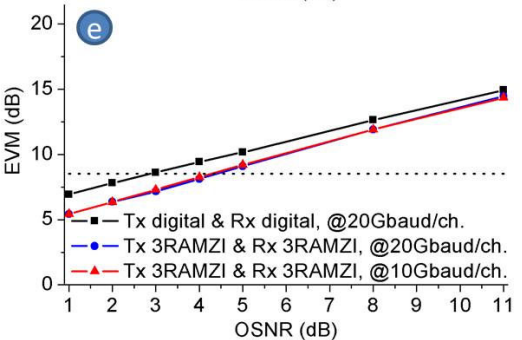

Fig. 2: (a) a scheme of Nyquist WDM system using 3RAMZI; (b) transmission performance comparison between digital and 3RAMZI Nyquist filter; (c) transmission performance comparison between digital and 3RAMZI matched filter; (d) comparison of required CMA filter taps between digital and 3RAMZI matched filter; (e) transmission performance of 3RAMZI used as both Tx Nyquist filter and Rx matched filter simultaneously and its scalability to arbitrary baud rate.

\section{Conclusion}

Using simulations, we have demonstrated the feasibility of using 3RAMZI as a high-performance optical Nyquist filter featuring a roll-off factor as small as 0.025 at the stopband suppression of $25 \mathrm{~dB}$, which is significantly steeper than most previous demonstrations of optical Nyquist filters. With its interleaver functionality 3RAMZI can be used for (de)multiplexing with the benefit of simplified system complexity. When used as a demultiplexer, it leads to a dramatic digital signal processing workload reduction for the receiver. A further investigation on its extended functionalities and experimental demonstrations using an on-chip realization will be performed in the near future.

\section{Acknowledgment}

This work is supported under the Australian Research Committee Laureate Fellowship scheme (FL13010041) and Centre of Excellence CUDOS (CE10001018).

\section{References}

[1] G. Bosco, A. Carena, V. Curri, P. Poggiolini, F. Forghieri, "Performance limits of Nyquist-WDM and CO-OFDM in high-speed PM-QPSK systems," IEEE Photon. Technol. Lett. 22(15), 1129-1131 (2010).

[2] G. Bosco, "Spectral shaping in ultra-dense WDM systems: optical vs. electrical approaches," Proceedings of OFC 2010, March 21-25, (2010).

[3] J. Schroder, M. A. F. Roelens, L. B. Du, A. J. Lowery, and B. J. Eggleton, "LCOS based waveshaper technology for optical signall processing and performance monitoring," Proceedings of OECC 2012, Busan, Korea, July 2012.

[4] R. Rudnick, A. Tolmachev, D. Sinefeld, O. Golani, S. Ben-Ezra, M. Nazarathy, and D. M. Marom, "Sub-Banded/Single-sub-carrier dropdemux and flexible spectral shaping with a fine resolution photonic processor," PD. 4.1, ECOC 2014, Cannes, France.

[5] C. G. H. Roeloffzen, L. Zhuang, C

[6] C. K. Madsen and J. H. Zhao, Optical filter design and analysis: a signal processing approach, Wiley, NY, 1999.

[7] L. Zhuang, D. Marpaung, M. Burla, W. Beeker, A. Leinse, and C. G. H. Roeloffzen, "Low-loss, high-index-contrast Si3N4/SiO2 optical waveguides for optical delay delay lines in microwave photonics signal processing," Opt. Express 19(23), 23162-23170 (2011).

[8] C. Zhu, B. Corcoran, A. V. Tran, and A. J. Lowery, "Nyquist-WDM with Low-Complexity Joint Matched Filtering and Adaptive Equalization,” DOI 10.1109/LPT.2014.2355203, IEEE Photon. Technol. Lett., 2014. 